\title{
Magnetic Field Dependence and Efimov Resonance Broadening in Ultracold Three-Body Recombination
}

\author{
Seth T. Rittenhouse \\ ITAMP, Harvard-Smithsonian Center for Astrophysics, Cambridge, MA 02138*
}

(Dated: November 21, 2018)

\begin{abstract}
We derive an analytic formula which describes the final bound state dependence in ultracold three-body recombination. Using an energy-dependent loss parameter, the recently observed broad resonance in an ultracold gas of ${ }^{6} \mathrm{Li}$ atoms $[1,2]$ is described quantitatively. We also provide an analytic and approximation for the three-body recombination rate which encapsulates the underlying physics of the universal three-body recombination process.
\end{abstract}

Over the last several years, the field of Efimov physics has generated a great deal of excitement $[3,4]$. Ultracold atomic gases provide a unique environment for studying these exotic states. Through the use of a broad FanoFeshbach resonance the s-wave scattering length can be tuned over several orders of magnitude [5-7]. Using these tools many universal behaviors associated with Efimov physics have been observed in both three- and four-body recombination processes [8-12], largely confirming many theoretical predictions (see Refs. [13-16] for some examples). The majority of these experimental studies have taken place using an ultracold gas of bosonic atoms.

Recently, a new class of Efimov state has emerged in a three-component degenerate Fermi gas of ${ }^{6} \mathrm{Li}$ atoms. For magnetic fields between 30 and $600 \mathrm{G}$, two resonances in three-body recombination have been observed $[1,2]$. The positions of these resonances corresponds to a single three-body bound state crossing the continuum at lower and then again at higher field strength. While the position of these resonances is fit very well by existing three-body recombination theory [17-19], the widths are not. The standard theory, in which it is assumed that the short range behavior of the three-body system is largely independent of the strength of the external magnetic field, predicts that the two resonances should have roughly the same width. The experimental evidence show a higher field resonance that is significantly broader than the first.

Three-body recombination occurs when two particles combine to form a dimer state releasing the resulting binding energy in the form of kinetic energy between the dimer and a third particle. It has been proposed by Wenz et al. [20] that the binding energy $E_{b}$ of the final dimer state is to blame for the observed discrepancy in resonance widths. The binding energy of the first several deeply bound two-body states in ${ }^{6} \mathrm{Li}$ have a strong magnetic field dependence [20]. By assuming a $1 / E_{b}$ dependence in the loss parameter they were able to find reasonable agreement with both resonances. The question still remains, however, as to where this energy dependence comes from and whether $1 / E_{b}$ is the correct form.

In this paper we present a simple mechanism that de- scribes the broadening of a three-body recombination resonance due to the magnetic field dependence of a deeply bound two-body state. We proceed by assuming that this precess can be described by a two-channel inelastic scattering process within the adiabatic hyperspherical representation, shown schematically in Fig. (1). The upper channel consists of three free particles scattering near threshold, while the lower channel consists of a tightly bound dimer with binding energy $\Delta$ and a free particle. The two channels are coupled at some small hyperradius $r_{0}$ by a non-adiabatic coupling $P$-matrix element:

$$
P_{i f}(R)=\left\langle\Phi_{f}(R ; \Omega)|d / d R| \Phi_{i}(R ; \Omega)\right\rangle_{\Omega},
$$

where $\Phi_{i(f)}(R ; \Omega)$ is the hyperangular channel function describing the initial (final) adiabatic channel and the matrix element is taken over the hyperangular degrees of freedom.

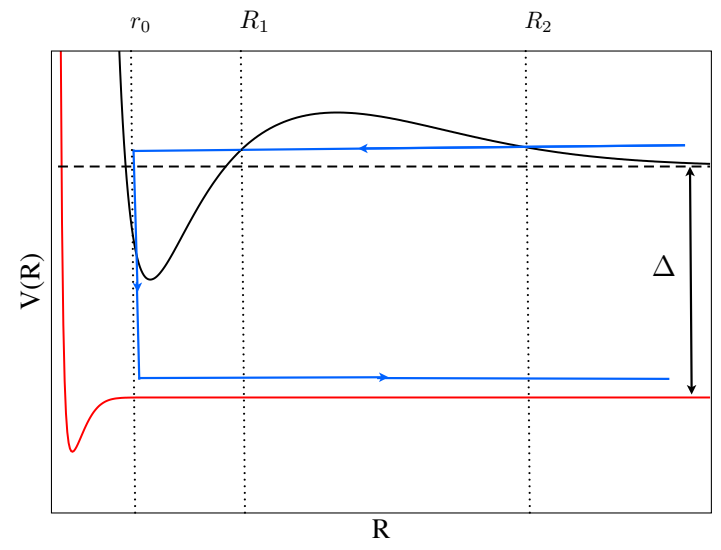

FIG. 1: (color online) A Schematic of the two-channel scattering process that controls threshold three-body recombination is shown.

Mehta et al. [16] showed that the inelastic scattering rate at the three-body threshold for this type of system can be described within the Wentzel-Kramers-Brillouin (WKB) approximation as

$$
K_{3}=\frac{9 \hbar^{5}}{4 m^{3} E^{2}} \frac{C e^{-2 \gamma_{W K B}} \sinh 2 \eta}{\cos ^{2} \phi_{W K B}+\sinh ^{2} \eta},
$$


where $m$ is the atom mass, $E$ is the initial asymptotic kinetic energy of the three atoms, and the constant $C$ is determined below. In Eq. (2) $\gamma_{W K B}$ is the WKB tunneling integral and $\phi_{W K B}$ is the WKB phase accumulated in the inner potential well of the upper potential, i.e.

$$
\begin{aligned}
& \gamma_{W K B}=\operatorname{Re}\left[\int_{r_{0}}^{R_{2}} \sqrt{\left[\frac{2 \mu}{\hbar^{2}}\left(V_{i}(R)-E\right)+\frac{1}{4 R^{2}}\right]} d R\right], \\
& \phi_{W K B}=\operatorname{Im}\left[\int_{r_{0}}^{R_{2}} \sqrt{\left[\frac{2 \mu}{\hbar^{2}}\left(V_{i}(R)-E\right)+\frac{1}{4 R^{2}}\right]} d R\right] .
\end{aligned}
$$

where $V_{i}(R)$ is the initial adiabatic potential which can be found using known methods (see for instance Ref. [21]) and $R_{1}$ is the outer classical turning point, and $E$ is the initial asymptotic kinetic energy of the three atoms. The extra $1 / 4 R^{2}$ in Eqs. (3) and (4) is due to the Langer correction. The proportionality constant $C \approx 300$ in Eq. (2) is extracted by comparing the $K_{3}$ in case when all three scattering lengths are equal to $a$, where $|a| \gg r_{0}$, to the known recombination rate for this case from Ref. [22]:

$$
K_{3}=\frac{\hbar 4590}{m} \frac{a^{4} \sinh 2 \eta}{\cos ^{2} \phi_{W K B}+\sinh ^{2} \eta} .
$$

In Eq. (2), $\eta$ is an imaginary phase which parametrizes the losses from the initial channel. The ratio of the outgoing to incoming probabilities in the initial channel [22] is given in terms of $\eta$ as

$$
\frac{\left|\Psi_{\text {out }}\right|^{2}}{\left|\Psi_{\text {in }}\right|^{2}}=e^{-4 \eta}
$$

The constants $\eta$ and $r_{0}$ are determined by short range physics and are usually used to fit the position and width of an Efimov resonance [17-19, 23]. For three-body recombination of distinguishable ${ }^{6} \mathrm{Li}$ atoms, assuming that $\eta$ and $r_{0}$ are fixed over the experimental range of magnetic fields, there is excellent agreement with the first three-body resonance [17-19] at approximately 130 G. A problem occurs however at the second resonance; the theory prediction is far narrower than the experimental results.

By assuming that the $P$-matrix element coupling the two channels is Lorentzian which peaks at $R=r_{0}$, the energy dependence of $\eta$ can be extracted. By considering the Landau-Zener transition probability, Clark [24] has shown that in this case the probability of making a non-adiabatic transition as the system passes through the transition region is given by

$$
\begin{aligned}
P_{n a} & =e^{-2 \pi \gamma}, \\
\gamma & =\frac{1}{\hbar v} \frac{\Delta}{8 P_{\max }},
\end{aligned}
$$

where $v$ is the characteristic velocity of the system at $R=r_{0}, \Delta$ is the energy separating the initial and final channels and $P_{\max }$ is the maximum of the $P$-matrix element. The velocity $v$ is determined by the short-range physics of the incoming channel while $P_{\max }$ will be determined by the small $R$ behavior of both the incoming and outgoing channel function $\Phi_{i}$ and $\Phi_{f}$.

While $P_{\max }$ cannot be determined exactly, we can make certain statements about its behavior. We assume that the short-range nature of the incoming channel is independent of the magnetic field over the range we consider. The outgoing final channel will be shifted overall, but the size and wave function of the final deeply-bound dimer state is relatively unaffected. Thus, both $P_{\max }$ and $v$ are independent of the final state energy $\Delta$.

By assuming that any non-adiabatic transition results in a three-body recombination event and the system must pass twice through the transition, on the way in and on the way out, the probability of remaining in the initial channel can be extracted:

$$
\frac{\left|\Psi_{\text {out }}\right|^{2}}{\left|\Psi_{\text {in }}\right|^{2}}=\left(1-P_{n a}\right)^{2} .
$$

Comparing Eqs. (6) and (8) gives a simple equation that can be solved for $\eta$ :

$$
\begin{aligned}
& \eta=\frac{1}{2} \ln \left[\frac{1}{1-\exp (-\beta \Delta)}\right], \\
& \beta=\frac{\pi}{\hbar v} \frac{1}{4 P_{\max }} .
\end{aligned}
$$

With this formula, the unknown short-range dependence of $\eta$ is encompassed in a single parameter $\beta$ which is independent of the binding energy $\Delta$. It is important to note that we have not increased the number of fitting parameters, we have merely shifted the fitting to the system dependent parameter $\beta$ instead of $\eta$. In cases where the final state energy does not strongly depend on the magnetic field, this extra dependence is not needed and $\eta$ can be used directly. However in cases where the final state has a strong field dependence, such as ${ }^{6} \mathrm{Li}$, the proposed parametrization of $\eta$ is more appropriate. In general a smaller binding energy leads to a larger loss parameter in Eq. (9), and a larger loss parameter leads to a broader resonance. Qualitatively, while the binding energy dependence in Eq. (6) is similar to the $1 / \Delta$ dependence assumed in Ref. [20], the detailed behavior is considerably different.

Figure (3) shows the recombination rates predicted from Eq. (2) in the threshold regime (incident energy $E=10^{-12}$ a. u.) compared to the experimental results of Ottenstein et al. [1] (red circles) and Huckans et al. [2] (blue squares). The dashed curve is found by assuming that $\eta$ is completely independent of the magnetic field strength. The solid curve is found by using Eq. (9) with the lowest dimer binding energy from Fig. (2b). Any 


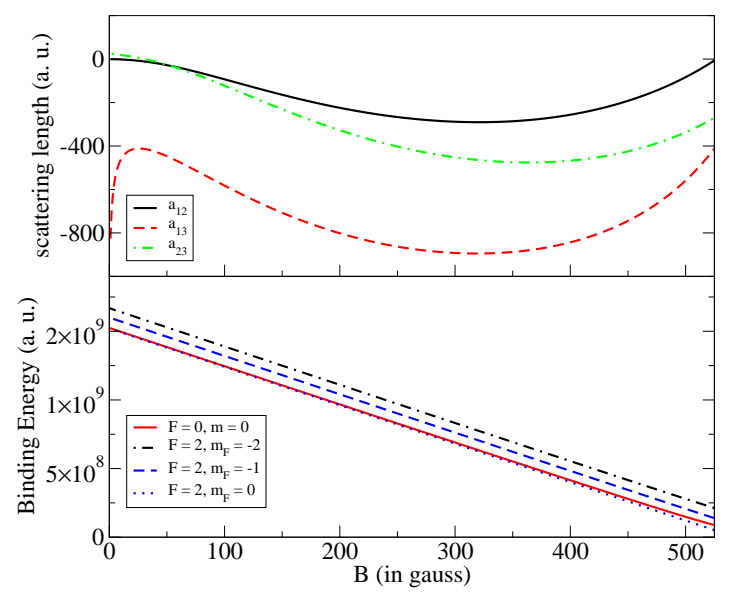

FIG. 2: (color online) (a) The three s-wave scattering lengths for ${ }^{6} \mathrm{Li}$ are shown in atomic units as a function of magnetic field. (b) The four smallest dimer binding energies from Ref. [20] are shown in atomic units as a function of magnetic field. The total nuclear angular momentum and projection are given, but are unimportant for the results in this work.

one of these four binding energies could have been used (with appropriate modifications to $\beta$ ), but because they all have similar magnetic field dependence, the results are nearly identical. The fits were found by setting $r_{0}=22$ bohr such that the the first resonance occurs at $130 \mathrm{G}$. The dashed curve was found by setting $\eta=0.05$ to fit the width of the first resonance at $B=130 \mathrm{G}$ using the data from Ref. [2]. The solid curve was found by choosing $\beta$ so that $\eta=\exp (-\beta \Delta)=0.05$ at the same $B$. Both of the predictions in Fig. (3) do an excellent job of describing the first resonance, while using the $\Delta$ dependent loss parameter from Eq. (9) is in astonishingly good agreement with Huckans et al. [2] and fairly good agreement with Ottenstein et al. [1].

The initial hyperradial potential used here was derived assuming that all scattering lengths were much larger than any short range parameters. The hyperradial potential used in Eqs. (3) and (4) were found by assuming zero range interactions, which is appropriate for scattering lengths much greater than the size of the two-bod interaction. In the case of ${ }^{6} \mathrm{Li}$, this size is given by approximately the Van der Waals length $r_{6} \approx 30 \mathrm{bohr}$. Because the smallest scattering length is not too much larger than this, we might expect small corrections to this due to non-universal behavior in the potentials. Even with this caveat, the qualitative agreement seen in Fig. (3) is remarkable.

While the treatment above does give good agreement with experimental data, it not necessarily convenient for quick comparison to experiment. Examining the three scattering lengths from Fig. (2a) shows that they differ by a factor of roughly 2 throughout the region in which the experimental data is taken. With this, we as-

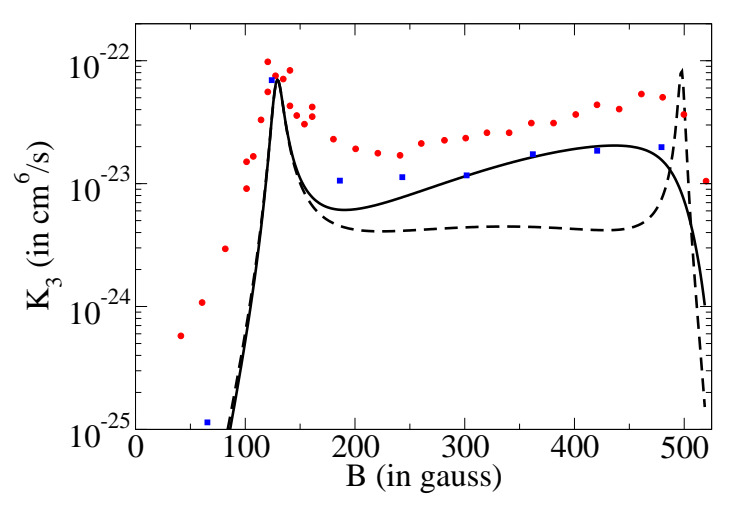

FIG. 3: (color online) The recombination rate constant predicted from Eq. (2) is shown for fixed $\eta$ (dashed curve) and for energy dependent $\eta$ (solid curve) as a function of magnetic field compared to experimental data from Ref. [1] (red circles) and Ref. [2] (blue squares).

sume that they are different enough to use the results of Ref. [23] which gives the incoming three-body hyperradial potential for three distinguishable particles with large s-wave scattering lengths in four regions:

$$
V_{i}(R)=\left\{\begin{array}{cc}
\frac{\hbar^{2}}{2 \mu} \frac{4-1 / 4}{R^{2}}, & \left|a_{l}\right| \ll R \\
\frac{\hbar^{2}}{2 \mu} \frac{4-1 / 4}{R^{2}}, & \left|a_{m}\right| \ll R \ll\left|a_{l}\right| \\
\frac{\hbar^{2}}{2 \mu} \frac{-s_{1}^{2}-1 / 4}{R^{2}}, & \left|a_{s}\right| \ll R \ll\left|a_{m}\right| \\
\frac{\hbar^{2}}{2 \mu} \frac{-s_{0}^{2}-1 / 4}{R^{2}}, & r_{0} \ll R \ll\left|a_{s}\right|
\end{array} .\right.
$$

Here $a_{l}, a_{m}$ and $a_{s}$ are respectively the largest, second largest and smallest scattering lengths in magnitude, and $s_{0}=1.006$ and $s_{1}=0.414$ are parameters which are determined by the universal potential in the limits where $R \ll\left|a_{s}\right|,\left|a_{m}\right|,\left|a_{l}\right|$ and $\left|a_{s}\right| \ll R \ll\left|a_{m}\right|,\left|a_{l}\right|$ respectively. It is not clear that these regimes exist for the scattering lengths shown in Fig. (2a), but we will proceed assuming that they do. By assuming that transitions between different universal behaviors have no significant contribution, the tunneling suppression and phase accumulation can be found using Eqs. (3) and (4):

$$
\begin{aligned}
e^{-2 \gamma_{W K B}} & \propto\left(a_{l} a_{m}\right)^{2}, \\
\phi_{W K B} & =s_{0} \ln \left(\left|a_{m}\right| /\left|a_{s}\right|\right)+s_{1} \ln \left(\left|a_{s}\right| / r_{0}\right), \\
s_{0} & =1.006 ; s_{1}=0.414,
\end{aligned}
$$

Inserting this into Eq. (2) yields an analytic formula for 


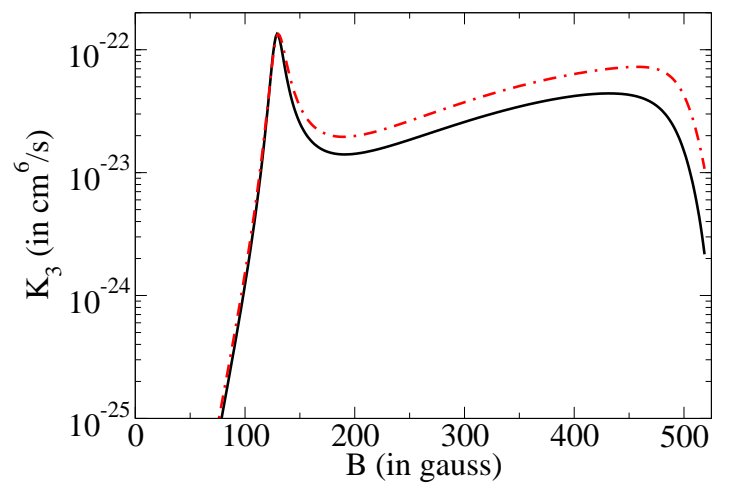

FIG. 4: The numerically calculated recombination rate constant from Fig. (3) (solid curve) compared to the analytic approximation from Eq. (13) is shown as a function of magnetic field strength.

the three-body recombination rate:

$$
K_{3}^{\text {univ }}=\frac{\hbar}{m} \frac{C^{\text {univ }}\left(a_{l} a_{m}\right)^{2} \sinh 2 \eta}{\cos ^{2}\left(s_{0} \ln \frac{\left|a_{m}\right|}{\left|a_{s}\right|}+s_{1} \ln \frac{\left|a_{s}\right|}{r_{0}}\right)+\sinh ^{2} \eta} .
$$

Figure (4) shows the results of this formula compared to the numerically obtained results in from Fig. (3). Both rates use the same $\Delta$-dependent loss parameter, while we have set $r_{0}=32.6 \mathrm{bohr}$ is Eq. (13) to fit the resonance position at $B=130 \mathrm{G}$. The constant $C^{\text {univ }}=1379$ has been chosen so that Eq. (13) agrees with the numerical results at the peak of the resonance. While the agreement may not be perfect it indicating that the analytic expression from Eq. (13) encompasses the majority of the relevant physics.

In summary, we have derived a simple expression that gives the loss parameter $\eta$ as a function of the binding energy of the final outgoing states. While this work focused on the three-body recombination resonances found in a three-component degenerate Fermi gas of ${ }^{6} \mathrm{Li}$ atoms, the method can be applied to other three-body systems. For instance in the case of three-body recombination to weekly bound dimers, the $P$-matrix and characteristic velocity $v$ are known, and Eq. (9) could be used to give a more complete description of the process. This parametrization of $\eta$ introduces no extra fitting parameters and gives excellent agreement with experiment. We have shown how a universal Efimov resonance can be broadened by the a field dependent binding energy of the outgoing final state. In general, a smaller dimer binding energy produces broader resonances when other parameters are held fixed. We have also given an analytic expression for the three-body recombination rate in a threecomponent degenerate Fermi gas of ${ }^{6} \mathrm{Li}$ atoms which provides reasonable agreement with the more complex numerical results. The energy dependent loss parameter was derived here using the intuition gained from the adiabatic hyperspherical method, but it is not limited to this approach and could be used wherever N-body recombination processes are parametrized by an imaginary phase. The process of determining the width of loss resonances based on knowledge of the dimer binding energy might also be turned around. One could use experimentally determined resonance behavior to find the field dependence of deeply bound final states.

The author would like to thank S. Jochim and H. R. Sadeghpour for useful discussions. Funding for this work was provided by a NSF through ITAMP at Harvard University and Smithsonian Astrophysical Observatory.

* Electronic address: \texttt\{srittenhouse@cfa. harvard.edu\}

[1] T. B. Ottenstein et al., Phys. Rev. Lett. 101, 203202 (2008).

[2] J. H. Huckans et al., Phys. Rev. Lett. 102, 165302 (2009).

[3] V. Efimov, Phys. Lett. B 33, 563 (1970).

[4] V. N. Efimov, Nucl. Phys. A 210, 157 (1973).

[5] S. L. Cornish et al., Phys. Rev. Lett. 85, 1795 (2000).

[6] M. Bartenstein et al., Phys. Rev. Lett. 94, 103201 (2005).

[7] S. E. Pollack et al., Phys. Rev. Lett. 102, 090402 (2009).

[8] S. Knoop et al., Nature Phys. 5, 227 (2009).

[9] G. Barontini et al., Phys. Rev. Lett. 103, 043201 (2009).

[10] M. Zaccanti et al., Nature Phys. 5, 586 (2009).

[11] F. Ferlaino et al., Phys. Rev. Lett. 102, 140401 (2009).

[12] S. E. Pollack, D. Dries, and R. G. Hulet, Science 326, 1683 (2009).

[13] B. D. Esry, C. H. Greene, and J. P. Burke, Phys. Rev. Lett. 83, 1751 (1999).

[14] P. F. Bedaque, E. Braaten, and H. W. Hammer, Phys. Rev. Lett. 85, 908 (2000).

[15] J. von Stecher, J. D'Incao, and C. Greene, Nature Physics 5, 417 (2009).

[16] N. P. Mehta et al., Phys. Rev. Lett. 103, 153201 (2009).

[17] E. Braaten et al., Phys. Rev. Lett. 103, 073202 (2009).

[18] P. Naidon and M. Ueda, Phys. Rev. Lett. 103, 073203 (2009).

[19] S. Floerchinger et al., Phys. Rev. A 79, 013603 (2009).

[20] A. N. Wenz et al., Phys. Rev. A 80, 040702(R) (2009).

[21] E. Nielsen et al., Phys. Rep. 347, 373 (2001).

[22] E. Braten and H.-W. Hammer, Phes. Rep. 428, 259 (2006).

[23] J. P. D'Incho and B. D. Esry, Phys. Rev. Lett. 103, 083202 (2009).

[24] C. W. Clark, Phys. Lett. A 70 (1979). 